\title{
$\underline{\mathbf{P}-103}$
}

\section{Triterpenes, Sesquiterpene and Alkaloids from Meiogyne Monosperma}

\author{
Asmah Alias ${ }^{1, *}$, Khalijah Awang ${ }^{2}$, Noraini Kasim $^{1}$, Nur Vicky Bihud ${ }^{1}$, Nurunajah AB Ghani and \\ Nor Hadiani Ismail ${ }^{1}$.
}

${ }^{I}$ Faculty of Applied Sciences, Universiti Teknologi MARA, 40450 Shah Alam, Selangor, Malaysia; ${ }^{2}$ Department of Chemistry, Universiti Malaya, 50603 Kuala Lumpur, Malaysia; E-mail: asmah_alias@yahoo.com

Meiogyne is a genus of flowering plants consists of 24 species belonging to the family Annonaceae. Only three Meiogyne species have been recorded in Malaysia which are $M$. cylindrocarpa, M. virgata and M. monosperma. From our previous work, M. virgata and M. cylindrocarpa were found to contain azafluorene alkaloid, aporphine alkaloids, oxoaporphine alkaloids and sesquiterpenes. This paper reports for the first time phytochemical study on M. monosperma which was collected from National Park, Pahang Malaysia. The stems of M. monosperma were extracted with dichloromethane for 8 hours by using Soxhlet extractor. After removal of the solvent, the crude extract was subjected to column chromatography fractionation with gradient elution using hexane:dichloromethane and dichloromethane:methanol solvent combinations. The fractions were monitored using thin layer chromatography and combined accordingly. Further purification steps involved centrifugal thin layer chromatography and preparative thin layer chromatography lead to isolation of four triterpenes, lupeol, campesterol, stigmasterol and $\beta$-sitosterol; a sesquiterpene, $\beta$-selinenol; and two alkaloids, liriodenine and lanuginosine. The structure of the compounds were established based on analysis of spectroscopic data including $1 \mathrm{D}$ and $2 \mathrm{D}$ NMR, FTIR, UV, MS and comparison with published data.

Keywords: Triterpenes, sesquiterpenes, alkaloids, Meiogyne monosperma. 